\title{
Análisis del índice de brecha de la intermediación en el Ecuador, durante los años 2016 y 2017.
}

\author{
Analysis of the gap index of the intermediation in Ecuador, during the \\ years 2016 and 2017. \\ Omar Gabriel Mejía Flores ${ }^{1}$., Erika Sonia Quiñonez Alvarado²., Flor María Lorena \\ Estrada Carrera ${ }^{3}$ \& José Luis Rivadeneira Pachec. ${ }^{4}$
}

\begin{abstract}
DOI: https://doi.org/10.33262/cienciadigital.v9i2.389

The Intermediation Gap Index (IBREI) in Ecuador is an indicator of the difference between the consumer price index and the producer price index. This is significant when studying the determinants of inflation, fundamentally those that could have speculative components of some items. The study of this difference and the issuance of official figures in this regard is not common in Latin America, Ecuador being one of the few countries that performs this process. However, during the years 2016 and 2017, there was a significant political change in the country that could have influenced the behavior of inflation, so the study of the behavior of this index through the deductive and analytical method is of vital importance . This study statistically reviews the IBREI data for the years 2016 and 2017, observing its stable behavior, despite noting also a significant difference in prices between the producer and the final.
\end{abstract}

Keywords: Psychosocial Risk, Mental Burden, Mental Fatigue.

\section{Resumen}

El índice de brecha de intermediación (IBREI) en el Ecuador, es un indicador de la diferencia existente entre el índice de precios al consumidor y el índice de precios al productor. Este, es significativo al momento de estudiar los determinantes de la inflación, fundamentalmente aquellos que pudieran tener componentes especulativos de algunos rubros. El estudio de esta diferencia y la emisión de cifras oficiales al respecto no es común en Latinoamérica, siendo Ecuador uno de los pocos países que realiza este proceso. Ahora bien, durante los

\footnotetext{
${ }^{1}$ Universidad de Guayaquil, Guayaquil Ecuador, omar.mejiaf@ug.edu.ec

${ }^{2}$ Universidad de Guayaquil, Guayaquil Ecuador, erika.quinoneza@ug.edu.ec

${ }^{3}$ Universidad de Guayaquil, Guayaquil Ecuador, flor.estradac@ug.edu.ec

${ }^{4}$ Alemán Humboldt, Guayaquil, Ecuador, jose.rivadeneira@alemanhumboldt.edu.ec
} 
años 2016 y 2017, se vivió un cambio político importante en el país que pudo haber incidido en el comportamiento de la inflación, por lo que el estudio del comportamiento de este índice mediante el método deductivo y analítico reviste una importancia vital. Este estudio, revisa estadísticamente los datos del IBREI para los años 2016 y 2017, observando su comportamiento estable, a pesar de notarse también una significativa diferencia de los precios entre el productor y el consumidor final.

Palabras clave: Riesgo Psicosocial, Carga mental, Fatiga Mental.

\section{Introducción.}

Uno de los indicadores de la salud económica de los países es el índice de inflación, la variación que tienen los precios a lo largo de la cadena de producción, distribución y comercialización, suele tener una incidencia fundamental en la estabilidad de los países, así lo afirma Tipan (2015) "la inflación es el fenómeno que presenta un alza generalizada de los precios; este se presenta como un problema para las economías, cuando sus resultados son muy elevados, a tal punto que afecta de forma negativa al bienestar de la sociedad." (p. 24).

Las decisiones de los agentes económicos se ven influenciadas por las cifras históricas de inflación, en tanto que como lo afirman Fuentes A. y Gimeno R. (2017) "las expectativas de inflación de los agentes son determinantes a la hora de analizar la evolución de muchas variables de decisión de empresas y familias. Además del valor promedio de las expectativas, también resulta relevante la probabilidad que los agentes asignan a escenarios extremos de inflación (como, por ejemplo, tasas muy elevadas o negativas), dado el impacto que estos tienen sobre la estabilidad y el crecimiento de la economía."(p. $1)$.

La autoridad monetaria de cada país es la encargada de emitir los índices de inflación, calculando las variaciones de precios en cada eslabón del circuito económico durante períodos de tiempo determinados, que suelen ser de un mes. Sin embargo, este fenómeno es diferente en cada eslabón del circuito económico y al mismo tiempo impacta de forma diferente según como sea medido.

De esta manera, es comúnmente aceptado el cálculo de inflación a través del Índice de Precios al Consumidor (IPC), el cual consiste en la revisión de los precios de una canasta de productos específica, por lo general los de mayor consumo para la población durante periodos mensuales y anuales. Además, se puede calcular la inflación en diferentes sectores, como la construcción, el productor, etc.

Ahora bien, una de las distorsiones más importantes en la formación de las expectativas de las personas en torno a la inflación, es la brecha que se genera en la formación de los precios a los niveles diferentes de la cadena de producción y comercialización. Quiere decir, que la inflación o la variación de los precios será diferente en los diferentes eslabones del circuito económico, y esta diferencia en la variación de los precios pudiera 
ayudarnos a hacer un mejor diagnóstico de la salud económica de ese país.

Se trata entonces de medir la variación de precios en los diferentes niveles económicos, para percibir si en ellos ha existido igual relación, pues suele ocurrir que la inflación en materias primas es mucho menos volátil que a nivel de comercialización de productos terminados, y esto por supuesto impacta y es diferente para los agentes intermedios y además nos indica el comportamiento de los agentes económicos.

Uno de estos índices es el elaborado por la Confederación Argentina de la Mediana Empresa (CAME) a través de su Índice de Precios de Origen y Destino (IPOD), indicando en su boletín electrónico "El IPOD es un indicador elaborado por CAME para medir las distorsiones que suelen multiplicar por varias veces el precio de origen de muchos productos agropecuarios en el recorrido desde que salen del campo y hasta que llegan al consumidor. Esas distorsiones son muy dispares según producto, región, tipo de comercio y época del año.

En general, las diferencias están determinadas por un conjunto de comportamientos, algunos especulativos, de diferentes actores del mercado."

Es de hacer notar, que no todos los países realizan la medición de este tipo de índices, y no siempre es la autoridad monetaria quien lo elabora. Tal es el caso de Argentina, donde es la organización empresarial quien se interesa en conocer este tipo de distorsiones. Sin embargo, en el Ecuador desde 1999 es el Instituto Nacional de Estadísticas (INEC) quien realiza el cálculo del Índice de Brechas de la Intermediación (IBREI), basados en una metodología estadística sencilla basada en la diferenciación y ponderación del Índice de Precios al Consumidor (IPC) con relación al Índice de Precios al Productor (IPP).

Al ser una institución gubernamental quien se encarga del IBREI, el interés es el de conocer el esquema de formación de precios en dicha economía, con especial atención en la inflación generada a causa de la intermediación de los bienes, tal como lo afirma Gordillo (2019) “con las brechas de precios obtenidas para la canasta de bienes específicos, compatibles del IPP e IPC, se forma una serie histórica de índices con la que se establece la evolución de la influencia inflacionaria proveniente de la Intermediación de Bienes." (p.2)

\section{Estado del arte}

El IBREI cobra importancia si queremos evaluar la economía ecuatoriana, en términos de los niveles de especulación y los determinantes propios de la inflación en diversos periodos de tiempo. A efectos de esta investigación, se busca observar el comportamiento de este índice particularmente durante los años 2016 y 2017, que significaron dos años de cambios políticos trascendentales para la nación.

En principio, se comenta la metodología propia del índice y se comenta su importancia, y luego, basados en la data proporcionada por el INEC, se realizan los análisis estadísticos que nos permitan analizar su comportamiento. A partir de esto, en estudios posteriores se 
podría generar inferencia y relaciones de otra índole entre el IBREI y el IPC, IBREI IPP, así como el IBREI y otras variables macroeconómicas.

El análisis de este índice, permite destacar su importancia e incentiva a privados y gobiernos para que generen esta clase de indicadores como componentes importantes de la inflación. De esta forma se adentra en los descriptores de una de las principales preocupaciones económicas de cualquier nación.

Sin embargo, son pocas las investigaciones al respecto, y estas, en el caso de Ecuador se centran en la comunicación organizacional interna y externa del INEC, con lo cual solo se está concluyendo sobre la importancia de dar a conocer el trabajo que realiza esta institución, tanto a lo interno como hacia la nación en general. Más no se ahonda en lo relacionado particularmente con el IBREI.

Por otra parte, son pocos los países e instituciones que muestran interés en medir la brecha de intermediación o la diferencia existente entre los precios al productor y los precios al consumidor.

Los ejemplos que pueden conseguirse son en Argentina, la CAME, que realiza la medición del IPOD, cuya metodología se orienta a determinar cuanto pagan de más los consumidores finales con relación a los intermediarios en puerta de finca o fábrica. Para ellos, es importante sobre todo el sector agrícola, que según su estructura de mercado debería tener menores márgenes de intermediación, de forma tal que sus productos fuesen preferidos en el mercado nacional, dado su precio. Sin embargo, no es una iniciativa estatal y no ha sido objeto de estudios científicos o académicos, resumiendo su relevancia al ámbito empresarial.

Igualmente, en países como México o El Salvador, algunas agencias de información y análisis económico dedican parte de su análisis a la diferencia entre las variaciones del índice de precios al productor y las variaciones del índice de precios al consumidor, como medida para explicar la inflación durante períodos particulares. Pero, la trascendencia no cubre al sector académico.

No significa esto que el estudio de estas diferencias y el cálculo de índices como el IBREI tenga poca importancia real. Ya que, en países como los latinoamericanos se puede observar un comportamiento especulativo en la cadena de distribución, que pudiera ser medido o revelado a través de mecanismos como la emisión de estos índices. Es inminente, que el sector privado se interesará en este tipo de estudios cuando, como en el caso argentino, tenga una fuerza productiva importante con intereses en la comercialización de sus productos dentro del mercado nacional. De lo contrario, queda a merced de la conveniencia de los Estados y la direccionalidad de sus instituciones, así como la importancia que puedan darle al estudio de las cadenas de distribución y comercialización y el impacto que el comportamiento de estas pueda tener en la inflación. 


\section{Método}

Para esta investigación se empleó el método analítico deductivo, usando como estrategia metodológica la revisión bibliográfica documental tanto física como digital disponible. Dado su alcance y analítico, se procuró la síntesis de los conceptos que componen el fenómeno estudiado.

Las fuentes documentales analizadas, variaron desde bibliografía técnico - científica para la elaboración de conceptos, hasta la revisión hemerográfica y legal, de manera que se observa la vigencia del tema.

Adicionalmente, se usó como herramienta metodológica el análisis estadístico, basado en la revisión de los datos obtenidos para el período de tiempo estudiado y generando las respectivas relaciones y conclusiones a partir de los resultados matemáticos obtenidos.

Blanco, 2011; Valledor, 2017 en Gamboa 2018, afirman que "es necesario un proceso de síntesis de la información obtenida de las acciones de la indagación empírica sobre la variable dependiente para arribar a conclusiones más generales relativas a ella." (p. 24). Para este fin, se empleó los valores mensuales del IBREI durante los años 2016 y 2017, presentados en la tabla 1, y a estos se les calculó las diferentes medidas descriptivas.

Tabla 1. IBREI Mensual años 2016 y 2017

\begin{tabular}{|c|c|c|c|c|c|c|c|c|c|c|c|c|}
\hline AÑOS MESES & ENERO & FEBRERO & MARZO & ABRIL & MAYO & JUNIO & JULIO & AGOSTO & SEPTIEMBRE & OCTUBRE & NOVIEMBRE & DICIEMBRE \\
\hline 2016 & 103,30 & 104,80 & 100,18 & 105,57 & 102,26 & 106,23 & 106,01 & 107,61 & 105,81 & 109,19 & 107,88 & 104,75 \\
\hline 2017 & 101,88 & 98,69 & 100,56 & 102,34 & 98,67 & 95,34 & 94,55 & 94,58 & 95,47 & 96,47 & 97,81 & 96,26 \\
\hline
\end{tabular}

Fuente: INEC

Es importante destacar que el IBREI es un índice y por lo tanto se expresa en porcentaje, representando la variación que ha tenido la brecha de intermediación o, dicho de otro modo, la diferencia existente entre los precios al consumidor y los precios al productor, de manera porcentual.

\section{Resultados}

Para el análisis de los datos referidos al IBREI de los años 2016 y 2017 se hace el cálculo de las medidas de estadística descriptiva, tanto de tendencia central como de dispersión, de manera que podamos evaluar la estabilidad del indicador a lo largo del periodo evaluado. Dentro de los cálculos realizados encontramos, el promedio o media aritmética, mediana, desviación estándar, varianza y curtosis de la muestra.

Es así como, cada una de estas medidas dará una representación válida del IBREI durante el periodo de estudio, y más allá, permitirá revisar el comportamiento de este índice durante el periodo de análisis.

La primera medida a tomar es la media aritmética o promedio. Como medida de tendencia 
central nos permite conocer cuál es el valor más representativo del IBREI durante los años 2016 y 2017, ya que como lo describen Levine, Krebhiel y Berenson (2015), "la media es la medida más común en la que todos los valores desempeñan el mismo papel. La media sirve como 'punto de equilibrio' del conjunto de datos (como el punto de apoyo de un balancín)". (p. 73)

Esta es una medida aritmética sencilla, que se calcula sumando todos los valores de la muestra y dividiéndolos entre el total de datos que la conforman. Tal como lo muestra la fórmula 1.

Fórmula 1. Media aritmética

$$
\bar{X}=\frac{x_{1}+x_{2}+x_{3}+x_{4}+\ldots+x_{n}}{n}
$$

Siendo x cada valor tomado por el IBREI desde enero de 2016 hasta diciembre de 2017 y el total de valores que conforman la muestra, que en este caso son 24 meses. En este caso, la media aritmética tiene un valor de 101,51\%.

El segundo valor obtenido es el de la mediana, medida que consiste en el valor que, luego de ordenados los datos lógicamente, divide la muestra en dos partes iguales. A tal efecto, Gamboa (2015) afirma que "la mediana siempre existe y es única, pero requiere que los datos puedan ser ordenados. Es de las medidas estadísticas más robustas, apropiada para un grupo pequeño de datos.” (p. 23)

Entonces la mediana, aunque puede ser calculada y formulada, no es en sí misma una medida aritmética, ya que depende estrictamente de que los datos puedan ser organizados. Para los valores del IBREI durante los años 2016 y 2017, la mediana tiene un valor de 102,07. Esto indica que al ser ordenados los 24 meses que representan la muestra, los datos del IBREI durante los años de estudio se agrupan de manera idéntica a los lados de dicho valor.

De igual manera, la moda es una medida de tendencia central no aritmética, la cual consiste en observar los valores que mas se repiten en una muestra. Así, podemos encontrar poblaciones en los que no existe moda, por cuanto todos y cada uno de los datos son únicos y, poblaciones que tienen más de un valor de moda, ya que existan en la misma frecuencia más de un dato específico. Para la observación de la moda, también es preferente establecer un orden lógico consecutivo de los datos.

Para el caso del IBREI durante los años 2016 y 2017, encontramos 3 modas, con valores que se repiten la misma cantidad de veces. Estos son $98,7 \%, 102,3 \%$ y $104,8 \%$.

Otro valor importante, que si bien no es de tendencia central, es una medida sencilla de variación en un grupo de datos, es el rango. Este se calcula luego de ordenados los datos, y sólo cuando estos son cuantitativos, restando el dato de mayor valor del de menor valor. 
El rango puede ser útil para conocer la dispersión de la muestra, en términos de la distancia existente entre los extremos de la muestra. En el caso del IBREI de los años 2016 y 2017 se obtiene un rango de 14,6.

Para la obtención de estas tres medidas fue necesario ordenar los datos de menor a mayor y para el caso específico de la moda, redondear eliminando el segundo decimal, a fin de obtener cifras iguales entre sí en algunos períodos. Tal como se muestra en las tablas 2 y 3.

Tabla 2. Datos del IBREI 2016 y 2017 ordenados secuencialmente de menor a mayor

\begin{tabular}{|r|c|c|c|c|c|c|c|c|c|c|c|c|}
\hline ITEM & 1 & 2 & 3 & 4 & 5 & 6 & 7 & 8 & 9 & 10 & 11 & 12 \\
\hline VALOR IBREI & 94,55 & 94,58 & 95,34 & 95,47 & 96,26 & 96,47 & 97,81 & 98,67 & 98,69 & 100,18 & 100,56 & 101,88 \\
\hline ITEM & 13 & 14 & 15 & 16 & 17 & 18 & 19 & 20 & 21 & 22 & 23 & 24 \\
\hline VALOR IBREI & 102,26 & 102,34 & 103,30 & 104,75 & 104,80 & 105,57 & 105,81 & 106,01 & 106,23 & 107,61 & 107,88 & 109,19 \\
\hline
\end{tabular}

Fuente: INEC con ordenación del autor

Tabla 3. Datos del IBREI 2016 y 2017 ordenados secuencialmente de menor a mayor con un decimal señalando los valores idénticos

\begin{tabular}{|c|c|c|c|c|c|c|c|c|c|c|c|c|}
\hline ITEM & 1 & 2 & 3 & 4 & 5 & 6 & 7 & 8 & 9 & 10 & 11 & 12 \\
\hline VALOR IBREI & 94,5 & 94,6 & 95,3 & 95,5 & 96,3 & 96,5 & 97,8 & 98,7 & 98,7 & 100,2 & 100,6 & 101,9 \\
\hline ITEM & 13 & 14 & 15 & 16 & 17 & 18 & 19 & 20 & 21 & 22 & 23 & 24 \\
\hline VALOR IBRE| & 102,3 & 102,3 & 103,3 & 104,8 & 104,8 & 105,6 & 105,8 & 106,0 & 106,2 & 107,6 & 107,9 & 109,2 \\
\hline
\end{tabular}

Fuente: INEC con resaltado del autor

Luego de las medidas estadísticas no aritméticas y de tendencia central, se pueden analizar grupos de datos calculando sus medidas de dispersión, tales como la desviación estándar, la varianza y la curtosis de la muestra determinada. Estos valores se orientan a determinar las características de la muestra con relación a la ubicación de los datos, por eso se les nombra de dispersión.

Una muestra estadística puede ser un conjunto o conglomerado de datos cercanos entre sí y adoptar valores similares, cuyos extremos no se alejan demasiado, o bien, pueden ser datos cuyos valores se distancien y varíen significativamente. Esta dispersión tiene incidencia en la interpretación de los datos, según el tipo de muestra y valores que nos encontremos analizando, ya que nos indican qué tanto varían los datos alrededor de la media. Tal como lo afirman Levine, Krebhiel y Berenson (2015) "en casi todos los conjuntos de datos, la mayoría de los valores observados quedan dentro de un intervalo de mas o menos una desviación estándar por encima y por debajo de la media. Por esa razón, conocer la media y la desviación estándar por lo menos dónde se agrupa la mayoría de los valores de los datos." 
Para el caso del IBREI de los años 2016 y 2017, fue calculado en primera instancia la varianza, ya que como sabemos esta es una cifra elevada al cuadrado, resultante de la suma de los cuadrados de los valores de la muestra, mientras la desviación estándar se deriva de esta. La fórmula 2 muestra cómo se obtuvo la varianza.

Fórmula 2. Varianza

$$
S^{2}=\frac{\sum_{j=1}^{n}\left(X_{j}-\bar{X}\right)^{2}}{n-1}
$$

De esta manera la varianza obtenida para los valores del IBREI durante los años 2016 y 2017 fue de 20,69

Entonces, para el cálculo de la desviación estándar puede optarse por sacar la raíz cuadrada de la varianza, de tal manera que se obtenga un valor en la misma dimensión que el resto de los datos. Así, la desviación estándar para el conjunto de valores del IBREI para los años 2016 y 2017 fue de 4,65\%.

Finalmente, la medida de concentración de los datos, denominada curtosis es calculada con la finalidad de conocer la forma en que los datos se agrupan alrededor de la media o valores centrales. El objetivo particular es conocer el tipo de distribución que tienen los datos y si el eje de simetría de dicha distribución es elevado o platicurtico.

Para el caso de los datos del IBREI de los años 2016 y 2017 la curtosis ha resultado en 1,32 ; con lo cual observamos que es un valor negativo que nos hace concluir que la distribución es platicurtica.

\section{Conclusiones.}

- Luego de la revisión estadística descriptiva de los datos del IBREI durante los años 2016 y 2017, se puede realizar un análisis detallado de este indicador en el periodo de estudio, relacionándolo además con la inflación y otras variables económicas del país.

- En primera instancia es importante destacar que los datos se encuentran dispersos alrededor de la media, al tener una curtosis negativa, la distribución de los datos se hace platicurtica, con lo que sabemos los valores no se encuentran altamente agrupados, sin embargo, si podemos intuir que se encuentran distribuidos de manera uniforme, con lo cual no esperamos grandes saltos entre los datos.

- Esto, lo constatamos cuando observamos un rango de 14,6; que nos indica que la diferencia entre el menor y el mayor valor tomados por el IBREI durante el periodo de estudio es de solo 14,6 puntos porcentuales.

- Todo esto, nos señala que la diferencia entre el índice de precios al productor y el 
índice de precios al consumidor es estable en el tiempo, aún cuando puedan existir variaciones significativas en la inflación. Con ello, podemos intuir que el comportamiento de la cadena de distribución y comercialización es uniforme y no se afecta con elementos de orden diferente a sus intereses económicos.

- Así mismo, podemos constatar estas afirmaciones cuando observamos que al trabajar con la data adecuada y el uso de dos decimales no hay una moda en la distribución de los datos, aun cuando todos los valores son cercanos y oscilan alrededor del 100\%, como más adelante lo analizamos con la media aritmética y la mediana.

- Los valores del IBREI durante los años de estudio se encuentran agrupados con una media de $101,51 \%$ y una mediana de $102,3 \%$, con lo que podemos intuir una distribución asimétrica, pero realmente con valores que al ser relacionados con la desviación estándar quedan oscilantes en un rango no mayor a 5\% en torno a la media. Esto quiere decir, que a través del tiempo la cadena distribución incrementa los precios en un promedio del $100 \%$ sobre los precios del productor, con lo que el consumidor ecuatoriano termina pagando el doble de lo que paga normalmente el distribuidor o comercializador a puerta de finca.

- De esta manera, pudiéramos evaluar que la inflación a nivel de productor es la mitad de lo que significa la inflación a nivel del consumidor, siendo las expectativas la única variable que pudiera generar una percepción diferente.

- Conforme a este estudio, la intermediación tuvo un costo medio de $100 \%$ durante los años 2016 y 2017.

\section{Referencias Bibliográficas}

Fuentes, A. y Gimeno, R. (2017). Indicadores sobre expectativas de inflación, basados en los precios de instrumentos financieros. Boletín Económico 3/2017. Recuperado de:

https://www.bde.es/f/webbde/SES/Secciones/Publicaciones/InformesBoletinesR evistas/ArticulosAnaliticos/2017/T3/fich/beaa1703-art22.pdf

Gamboa, M. (2017). Estadística aplicada a la investigación educativa. Revista Dilemas Contemporáneos: Educación, Política y Valores. V (2). Articulo 5. Recuperado de: https://dilemascontemporaneoseducacionpoliticayvalores.com

Gordillo L. (2015). Metodología del Indice de Brechas de la Intermediación (BRE - I). Recuperado de: http://www.ecuadorencifras.com

Levine D., Krehbiel T. \& Berenson M. (2015). Estadística Descriptiva. Pearson Educación. Mexico.

Tipán, B. (2015). Análisis del Gasto Público sobre la Inflación en la Economía Ecuatoriana en el Período 2000 - 2012. Universidad de Cuenca. Recuperado de: http://dspace.ucuenca.edu.ec/handle/123456789/22333 


\section{Para citar el artículo indexado.}

Mejía O., Quiñonez E., Estrada F.\& Rivadeneira J. (2019). Análisis del índice de brecha de la intermediación en el Ecuador, durante los años 2016 y 2017. Revista electrónica Ciencia Digital 3(2), 264-274. Recuperado desde: http://cienciadigital.org/revistacienciadigital2/index.php/CienciaDigital/article/view/389 $\underline{1837}$

\section{LCiencia}

El artículo que se publica es de exclusiva responsabilidad de los autores y no necesariamente reflejan el pensamiento de la Revista Ciencia Digital.

El artículo queda en propiedad de la revista y, por tanto, su publicación parcial y/o total en otro medio tiene que ser autorizado por el director de la Revista Ciencia Digital.
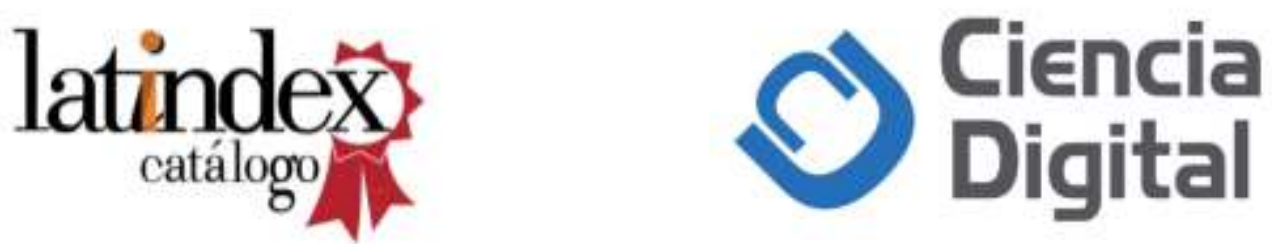